\title{
Low-Cost Bluetooth-Arduino Hover Control Design of a Quad Copter
}

\author{
Supantha Mandal ${ }^{1}$, Suraj Kumar $\mathrm{Saw}^{2}$, Karan $\mathrm{Shaw}^{3}$, Arunava Kabiraj Thakur ${ }^{4}$, \\ Varsha Seth $^{5}$, Puja Singh ${ }^{6}$ \\ Department of Electronics \& Communication Engg., Department of Computer Science Engg. \\ National Institute of technology Arunachal Pradesh India \\ St. Mary's Technical Campus Kolkata, India ${ }^{1,3,4,5,6}$
}

\begin{abstract}
This paper presents bluetooth controlled arduino integrated with collision detection, avoidance and contol features which provides the unique ability of flight stablization, hover contol and landing features in any terrain.The extremely sophistcated design provides very good hover contol of the design.The design integrated with ultrasonic sensors with in built accelerometer controls the speed and avoid collision with any obstacle detected in the flight of the quadcopter.The parts used in the design are of low cost making the design economical which makes it ideal for production on industrial scale which will aid in many applications like collecting data, spying and in miltary operations.In this research article a entire system is designed and inplemented in which flight,hovering is stably controlled based on feedback from ultrasonic sensors and visual feedback.A low cost robust design has been implemented with advanced features which makes it very unique and attractive for commercial production.
\end{abstract}

Keywords: Unmanned aerial vehicle (UAV); personnel digital assistant (PDA); Degree of Freedom (DOF); Revolutions per minute (RPM); Graphical user Interface (GUI).

\section{Introduction}

A unmanned aerial vehicle also known as quad copter is governed by flight dynamics in which various forces of nature and mechanical forces for hovering is taken into consideration to ensure smooth propelling and hovering of the vehicle when it is airborne. Its working principle is based on variation of spin RPM of the 4 servomotors to stabilize and control lift and torque. The thrust from the four servomotors attached diagonally at the design provides a major role in maneuvering and to ensure the quad copter airborne. Precise angle handling is essential to perform the flying routines and to maintain complex trajectory governed by laws of physics. Our design serves as a solution to demonstrate how the spin of the servomotors to achieve high degree of angular orientation giving the design precise control from the user side to achieve standard flight operations like hovering at a particular altitude, take off from ground to avoid any obstacle to prevent collision and ensure smooth landing[1] . Quad copters are like conventional small helicopters but simpler in mechanical design and achieve high degree of flight stabilizing and maneuverability. The driving forces like torque, roll, pitch and yaw which are controlled by 4 pitch propellers in the form of servomotors connected at the quad copter ensure flight stability. Modern day quad copters are integrated with sensors which has increased their popularity tremendously in field of surveillance, data collection from aerial platform and in military applications reaching a benchmark of excellence in modern day of electronics and communication engineering fused with the advanced aspects of aerodynamics. The earlier design of quad copters was unstable due to non linear dynamics. The modern design are provided with 6 degree of freedom which give the user precise control of the unmanned aerial vehicle from a wireless module by radiofrequency control or any sort of wireless medium .In existing literature many works have been done on the analysis and implementation of the quad copter for various aspects like photography and disaster management and military application[2].In our paper we have designed a arduino integrated with flight connector board with inbuilt accelerometer to control the speed of the 4 brushless motors and to stabilize the flight by remote controlling from a mobile device having Bluetooth as the controlling medium .Our user end equipment is equipped with graphical user interface developed with java language to control the quad copter flight and landing. Our design includes arduino uno has the which is the central unit of the design integrated with flight connector board which issues command for controlling the speed of the motors which are having mechanical shock absorbers to prevent any damage to quad copter while landing. The collision and detection protocol has been incorporated and well executed by using ultrasonic sensors which prevents collision and sends the signal to the user mobile of obstacle detection. The quad copter flight direction is easily controlled with the alignment of the mobile or PDA in which the controlling software is used. It behaves like a joystick thus controlling the direction flight in any direction when the mobile is tilted or aligned in a particular direction. The most attractive feature is the voice recognition command platform in the mobile which take takes 
the command in voice and executes the motion of the quad copter to turn left, right, up, down which makes the design very useful as it has full control from the user end by input signal delivered in any form whether it is manual mode or voice mode

\section{II. proposed model}

Our proposed model is a very highly efficient, low cost, low power consuming quad copter controlled by voice module which includes speech processing from user side to control and align the aerial vehicle in whatever direction we need. Our model can be manually operated from a remote mobile device by wireless medium Bluetooth which also includes control by tilting the mobile in any degree which gives all around degree of freedom to the quad copter when it is airborne. The graphical user interface develop by java portal is extremely attractive with enhanced visual platform showing all control directions of the quad copter resulting in high degree of control and precision. Our design is very unique because of integration of arduino Uno with very light weight flight control board which issues the control commands. Every module and peripheral interface is well synchronized in its mode of operation. There is high degree of speed control of motors attached at the quad copter with the use of inbuilt accelerometer which thus controls the flight dynamics, controls the trust, torque and other aerodynamic features ensuring smooth take off and landing. Our design includes ultrasonic sensors for obstacle detection, and provides feedback to the controlling device like mobile/PDA in which the graphical control interface is installed thus avoiding collision and changing of path is very easy in our design. We have used a low cost Bluetooth module which enables dual mode of communication of control data between the controlling station and the quad copter. We have used arguing Uno board which is easily programmed by in system programming in the integrated development environment which is the most enhanced feature of arguing in simple c language preventing the hassles of machine level coding. The arduino Uno is extremely portable which has a very powerful microcontroller ATmega328 which enables very easy coding and execution of commands. The arduino uno is interfaced with ultrasonic sensor module, flight controller board and Bluetooth module. The Bluetooth module is interfaced with the controlling station mobile or PDA which controls the maneuvering and hovering of the quad copter resulting in a high efficient design with enticing features.

\section{Literature survey}

Nowadays, extensive study on quad copters has attracted students and researchers due to its broad scope of unmanned aerial vehicle applications. The most popular kind of UAV is Quad copter based on arduino uno. The research related to Quad copter covers the various aspects of electronics and aerodynamics for proper design, control, stability, communication, collecting data from remote and hazardous areas with incorporation of collision detection and avoidance features.

The use of graphical user interface developed with various software's like lab view, processing has been very popular for controlling of Quad copter has been widely used globally nowadays. Reference [5] study is based on designing GUI control of quad copter based on software genetic algorithm. The GUI developed is multi button and multi remote control. Reference [6] learned GUI control of UAV for testing, control objectives. The analysis and understanding is based on genetic algorithm. Reference [7] investigated GUI for easy control, handling and detection of the bottle for leakage issues. Reference [8] focused their experimentation on flying quad copter with 3 degree of freedom with altitude control features and precise control of the unmanned aerial vehicle. Reference [9] indicates the development of non linear control of the UAV and development of non linear model of the quad copter with six degree of freedom. The nonlinear model of flying robot is based on the principle formulated and derived by Newton-Euler .The quad copter Model derivation consists of getting the equations of 3 dimensional motion of quad copter and enforce approximate actuation forces equations through aerodynamic modeling Coefficients. Reference [10] is based on control coordination of Quad copter by visual tracking and perception using camera. In this article, visibility is enhanced by use of active markers. Reference [11] worked on Quad copter intelligent fuzzy controller. An entire analysis and modeling is based on fuzzy controller principles. The inputs of the UAV are the expected values of the, pitch, height, roll and yaw. The output desirable are powers obtained at 4 rotors of the quad copter. Efficiency of this intelligent control strategy is determined by software simulation. References $[12,13]$ research is based on analysis of the dynamic characteristics of a Quad copter and determination of PID controller performance. The article is based on architecture of Quad copter. This paper also aims at controlling the posture of the UAV with 6 degree of freedom.

Table 1: The Comparitive Study Of Various Technoligies Used

\begin{tabular}{|l|l|l|l|}
\hline Sequence number & Research Title & Merits & Demerits \\
\hline$[12]$ & $\begin{array}{l}\text { 3-DOF attitude } \\
\text { control free-flying vehicle }\end{array}$ & $\begin{array}{l}\text { Simplicity of } \\
\text { Controller design. }\end{array}$ & $\begin{array}{l}\text { Restricted degree of freedom } \\
\text { allowing only 3 -DOF }\end{array}$ \\
\hline$[13]$ & $\begin{array}{l}\text { Nonlinear model and } \\
\text { nonlinear control }\end{array}$ & $\begin{array}{l}\text { Compensation of pitch } \\
\text {,yaw angles, controlling }\end{array}$ & $\begin{array}{l}\text { exclusive design for balancing } \\
\text { during }\end{array}$ \\
\hline
\end{tabular}




\begin{tabular}{|l|l|l|l|}
\hline & $\begin{array}{l}\text { Strategy for a 6-DOF } \\
\text { Quad copter aerial } \\
\text { Robot }\end{array}$ & $\begin{array}{l}\text { initial and } \\
\text { error, stabilize roll and } \\
\text { errors and } \\
\text { maintain them at zero. }\end{array}$ & hovering motion of Quad copter \\
\hline$[14]$ & $\begin{array}{l}\text { Control of } \\
\text { Quad copter by visual } \\
\text { tracking using stereo } \\
\text { camera }\end{array}$ & $\begin{array}{l}\text { Easy set up and portability } \\
\text { of the design }\end{array}$ & $\begin{array}{l}\text { Photosensitive to low intensity } \\
\text { light and not very useful } \\
\text { at high illumination area }\end{array}$ \\
\hline$[15]$ & $\begin{array}{l}\text { Intelligent fuzzy } \\
\text { controller of } \\
\text { Quad copter }\end{array}$ & $\begin{array}{l}\text { small overshoot and faster } \\
\text { dynamic response of } \\
\text { of fuzzy controller }\end{array}$ & Complexity of controller design \\
\hline
\end{tabular}

Our aim is to understand the Quad copter structure and study basic mathematical modeling, design of the quad copter with analyzing the individual components and to test them to ensure proper operation of the quad copter to enhance its features and make sure it is stabilized in its flight mode.

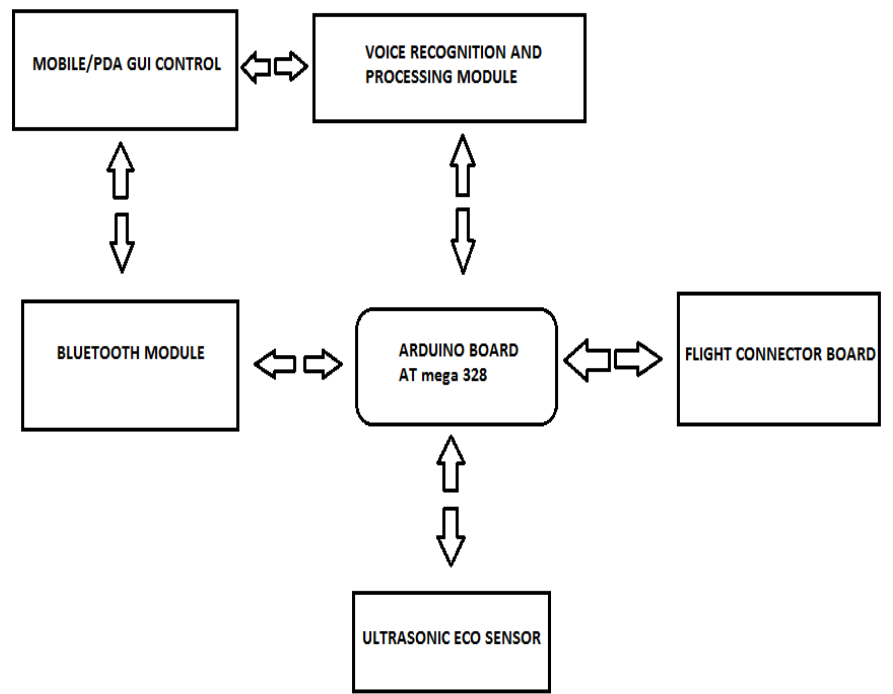

Fig:1 Block diagram of Quad copter

The above block diagram illustrates the hardware components modules which are integrated with each other providing us a robust, compact, portable design. The block diagram consists of arduino nano board module acting as central unit connected to four modules (ultrasonic sensor module, flight connector board, Bluetooth module, voice recognition and processing module).The mobile GUI controller module is attached with Bluetooth and voice recognition module for control signal transmission which controls the operation of the quad copter and flight direction which is depicted in the above block diagram.

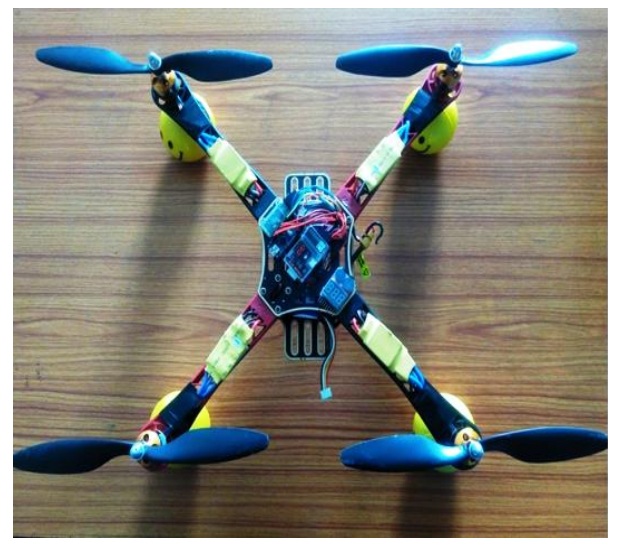

Fig: 2 Hardware assembled design of Quad copter

Fig.2 shows the hardware assembled design of quad copter which includes two diagonal arms integrated with four dc motors which creates balanced flight condition. Enabling smooth take off and landing maintain the stability of aerodynamics parameters. 


\section{Quad copter movement mechanism}

Quad copter can be presented and described as a small aerial vehicle with four propellers integrated to rotors which are located at the cross frame. The objective for fixed pitch brushless rotors is to control the vehicle motion dynamics. The four rotors speeds are independent and hence roll; pitch and attitude, yaw of the vehicle can be control with ease. UAV has four inputs force with the thrust that is generated by the propeller that connects to the brushless rotors. Taking off is the movement of UAV that lifts the quad copter up from ground to the required hover position? Landing position is opposite of take off position. Landing motion is kept in check and control by incrementing and decrementing of the speed of four rotors simultaneously which indicates the change of vertical motion of quad copter. The motion in Forward (backward) is controlled by incrementing and decrementing the speed of (front) rear brushless Rotor. Decrementing (incrementing) the (front) rear brushless rotor speed simultaneously will have control over the pitch angle of the Quad copter. For right and left motion of quad copter, it can be controlled by making change in the UAV yaw angle. Yaw angle of yaw can be controlled by increase and decrease in counter-clockwise brushless rotors speed integrated with flight connector board. Static position or hovering of Quad copter is implemented by two pairs of brushless rotors which are rotating in counter-clockwise and clockwise directions respectively with equal speed. By the two rotors moving in counter-clockwise position and clockwise direction, the reaction torque total sum is zero and hence this dynamics allows control of Quad copter in hovering position.

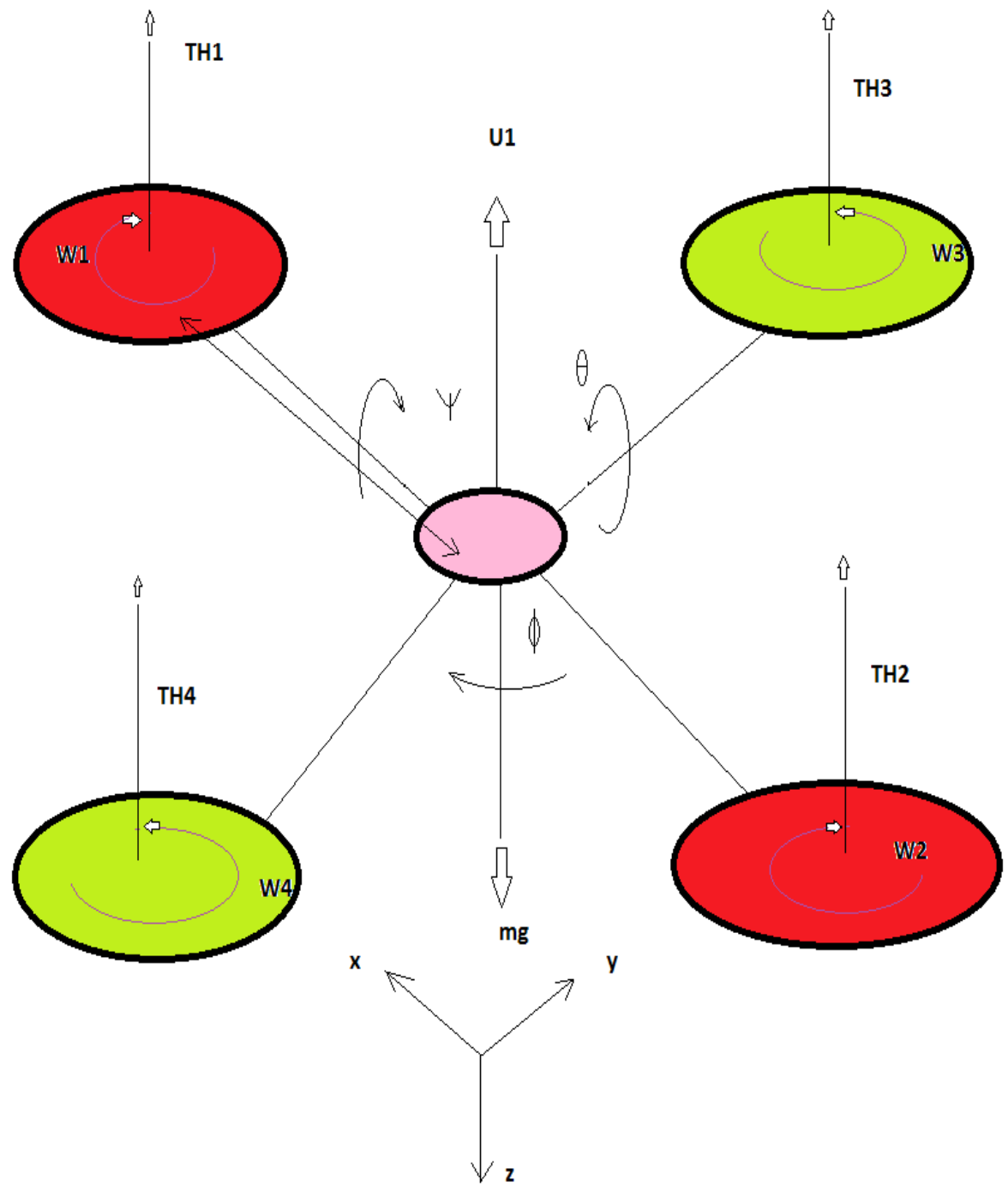

Fig: 3 Schematic diagram UAV 


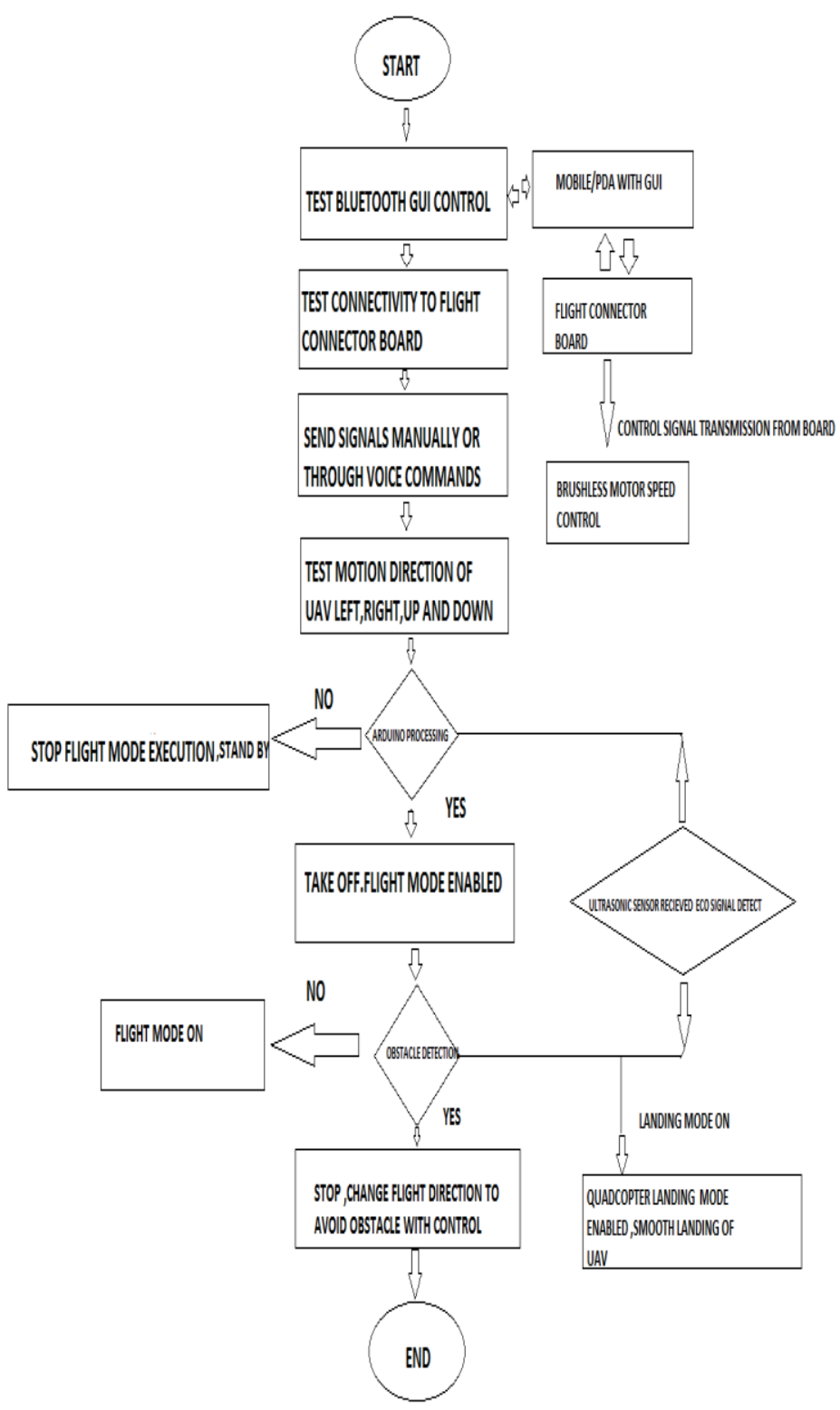

Fig:4 Flow chart of Quad copter operation

The above flow chart explains the operation of quad copter in sequential manner. The flow chart indicates the initialization and execution of UAV flight .The quad copter on activation by GUI Bluetooth signal tests connectivity and control to flight connector board. When connectivity is successfully established with the controlling device, quad copter becomes ready for taking its flight .Bluetooth connectivity is tested first and then on transmission of signals for executing take off makes the UAV airborne with control of flight directions by both manual graphical user mode or voice command module incorporated in the mobile. The quad copter is equipped with flight changing direction protocol when it comes across obstacles. The quad copter altitude and flight dynamics parameters like thrust, yaw, roll is adjusted by remote controlling from mobile by use of Bluetooth which leads to high precision of flight dynamics. When it encounters an obstacle, it alerts the user by use of feedback signal and changes its direction when it receives the next signal. It goes in landing mode when commands are given from user side to ensure smooth landing of the UAV.

\section{A. Bluetooth Module}

\section{Components}

v2.0 +Enhanced data rate Bluetooth module for $2 \mathrm{Mbps}$ and 3Mbps modulation modes has been deployed in our design .High speed operation and accuracy with full, scatter net support Pico net support is achieved by this module. Easy interfacing with the microprocessor ATMEGA 328 is our greatest advantage achieved with minimum number of external components with added feature of very lower power consumption 
making the Bluetooth module a full compliant, reliable system for data and voice communication. Low latency and low power for exchanging data over short distances is an attractive feature of the module. Gaussian frequency shift key modulation enables transmission of data over short distances in our quad copter design.

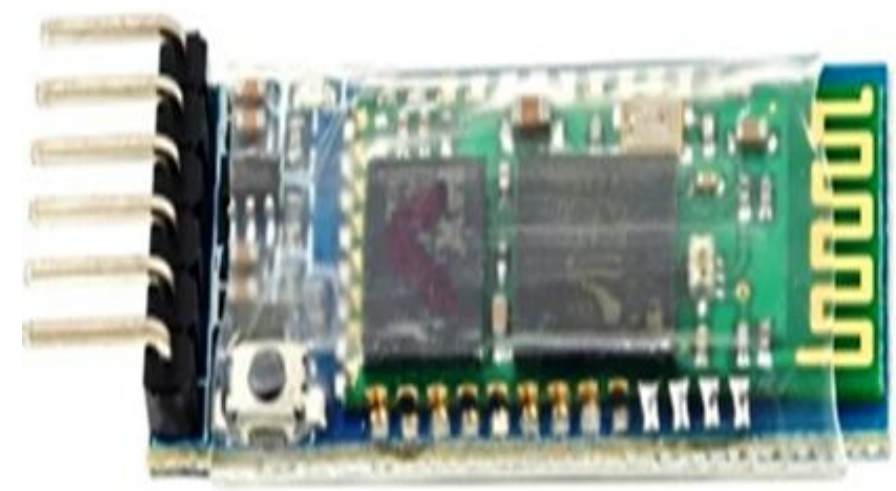

Fig5: Bluetooth module

\section{B. Arduino Nano}

In our design we have used arduino nano which is a complete, small, portable board which can be easily be interfaced with other modules of quad copter .It operates with very small voltage of 5 v.It has 14 digital input output pins of which 6 provides PWM output along with 8 analog input pins. It has $32 \mathrm{~kb}$ of flash memory which helps in easy storage of data and efficient processing of signals. Less weight and small dimensions are attractive features which makes the board very useful.ATmega328 or ATmega168 is powerful microprocessor used in arduino nano which process all instructions and swiftly carries all mathematical and logical operations. The most convenient feature is its easy in system programming which enables easy coding in c/java language overcoming the complexity of assembly level coding.Arduino nano comes with very useful software serial library which enables easy serial communication on any of the digital pins of arduino nano. The integrated development environment which is the novel feature of arduino nano helps to create graphical designs and interfaces with ease when integrated with graphical designing software's like lab view and processing 2 software.

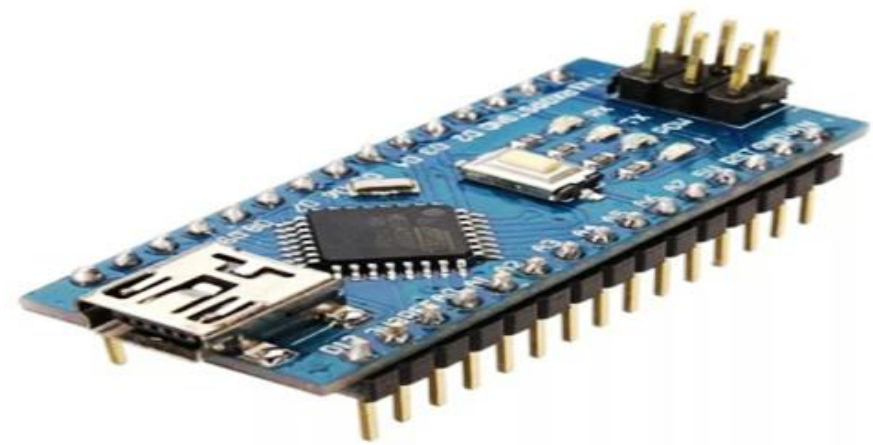

Fig: 6 Arduino nano

\section{Copters IB0852 Brushless DC Motor}

In our design we have used 4 brushless motors operating with 1300kv which are diagonally attached in the flight connector board. The dc motors are compact, sturdy and robust in size which provides maximum thrust of 920 gms.It is small, portable and weighs only $53 \mathrm{gms}$.High electrical efficiency is obtained with high quality precise parts making it suitable for medium to large size quad copters, octacopters and hexacopters. The composition is of hard steel supported by sealed dual bearings. This brushless motor have propeller of $5 \mathrm{~mm}$ diameter and motors can be easily run by 2 to 4 lithium batteries making it ideal choice for operation of the flight connector board creating adequate thrust to keep UAV airborne giving good control on hovering and altitude control of quad copter. 


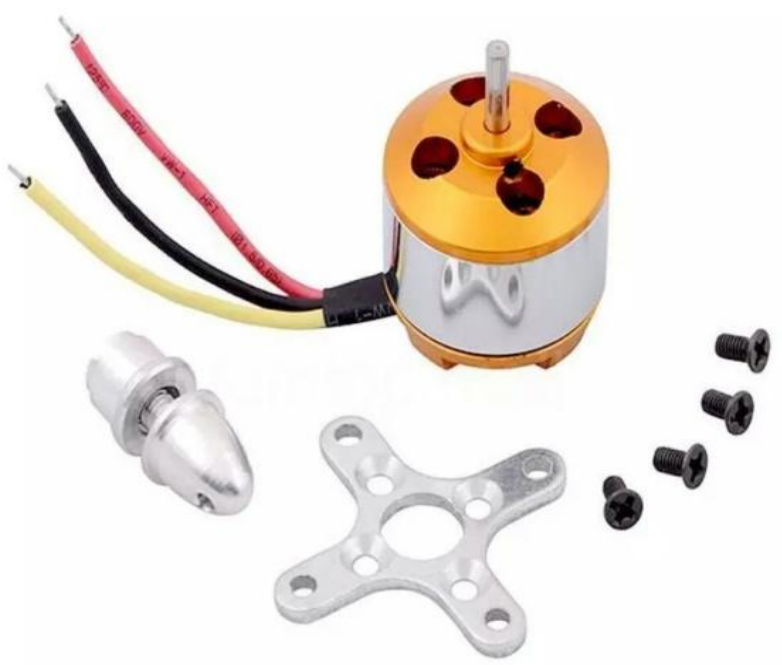

Fig: 6 brushless DC Motor

\section{30A BLDC ESC Electronic Speed Controller:}

An electronic speed control is controlling circuit for varying speed of electric motor, control direction and to act as dynamic brake. They are widely used on radio models often integrated with brushless motors for providing 3phase electric power. It acts as single unit used for throttle control and can be incorporated in a receiver. Our ESC varies the direction and speed of IB0852 brushless dc motor. The low voltage and power requirement is very unique feature of the ESC.Its weight is only $23 \mathrm{gm}$ with small dimensions and operating current 30A makes it a ideal choice for our design.

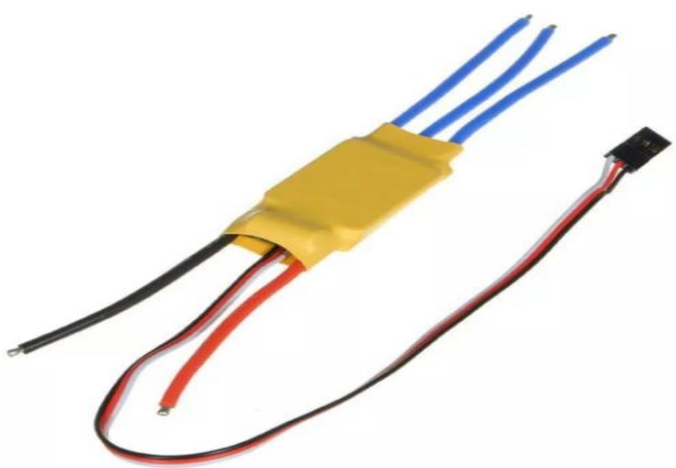

Fig:7 Electronic speed controller

\section{E. Lipo battery charger( Imax B3 Pro Compact charger):}

A battery charger puts energy in secondary cell by virtue of passing current through it. The protocol for charging depends on the type and size of battery. High tolerance for overcharging, recharging are unique characteristics of battery chargers. Our lipo battery charger works on ac input voltage of $(110 \mathrm{v}-240 \mathrm{v})$ with balance charge current $850 \mathrm{~mA}$.Its plastic packaging with maximum power rating of $10 \mathrm{~W}$ makes it ideal choice for the operation of quad copter.

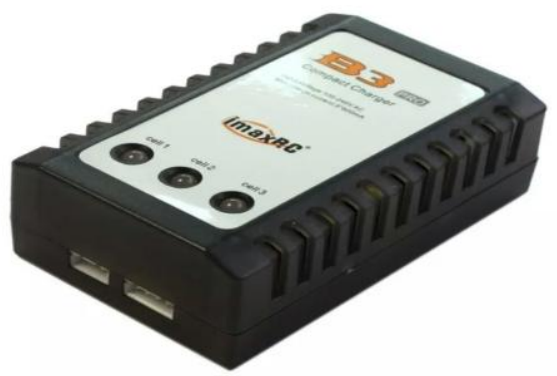

Fig:8 battery charger 


\section{F. QQ Superflight multi rotor controller}

The QQ Super multi-rotor flight controller has inbuilt 3 axis acceleration sensor and 3 axis gyroscope incorporated with stability function which provides a super smooth stable and controlled flight performance. All general receivers including Futaba S.BUS, DSMX are compatible and supported by the flight controller. Multi-rotor type and receiver type are selected easily via tiny DIP switches with extremely easy using set button. It has good sensivity once calibrated with only one small adjuster . The unit includes 2 $\mathrm{x}$ double sided mounting tape, $4 \mathrm{x}$ male to male connectors with a screwdriver for making adjustments making it ideal choice for our design .It unique support for multi-rotors with up to 6 motors and 4 configuration types support makes it a good unique choice. It is light weight, compact, and portable which suffice the design which helps in flight stability and control of aerodynamic parameters.

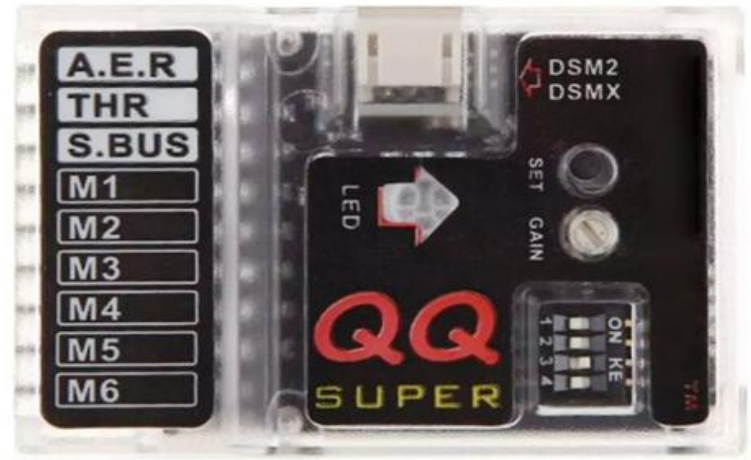

Fig: 9 Super flight Controller

\section{G. Quadcopter frame 450mm:}

The Q450 V3 is a well planned $450 \mathrm{~mm}$ quad frame built with high quality materials. The main frame is made from glass fiber while ultra durable polyamide nylon is used to construct the arms. It includes integrated PCB connections for direct soldering. This prevents the need for a power distribution board which makes the board light and portable. It comes with pre-threaded brass sleeves for frame stability .A great feature of this frame includes large mounting tabs at rear and front end of the main frame bottom plate for attaching cameras or other electronic sensing modules. This makes for a great way to take aerial video or fly FPV without the need to add any additional mounting brackets. The Q450 colored arms makes are appropriate for flight orientation. It enables you to fly in the right direction without the need for different colored props. These all unique features make this frame suitable for integration with the design and provide high reliability.

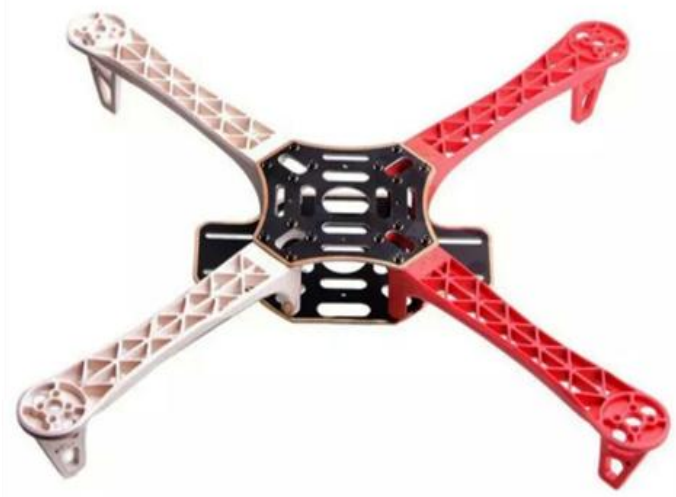

Fig:10 Quadcopter frame

\section{Mathematical equations governing Quad copter}

$\mathrm{U} 1=$ sum of the thrust of each motor

Th1 $=$ thrust generated by front motor

$\mathrm{Th} 2=$ thrust generated by rear motor

$\mathrm{Th} 3=$ thrust generated by right motor

Th4= thrust generated by left motor

$\mathrm{m}=$ mass of Quad copter

$\mathrm{g}=$ the acceleration of gravity 
$1=$ the half length of the Quad copter

$\mathrm{x}, \mathrm{y}, \mathrm{z}=$ three position

$\theta, \phi, \varphi=$ three Euler angles representing pitch, roll, and yaw

The dynamics formulation of Quad copter moving from landing position to a fixed point In the space is given as:

$$
R x y z=\left[\begin{array}{ccc}
C \phi C \theta & C \phi S \theta S_{\psi}-S_{\phi} C_{\psi} & C_{\phi} S_{\theta} C_{\psi}+S_{\phi} S_{\psi} \\
C_{\phi} S_{\theta} & S_{\phi} S_{\theta} S_{\psi}+C_{\phi} C_{\psi} & S_{\phi} S_{\phi} C_{\psi}-C_{\phi} S_{\psi} \\
-S_{\theta} & C_{\theta} S_{\psi} & C_{\theta} C_{\psi}
\end{array}\right]
$$

Where,

$\mathrm{R}=$ Transformation matrix

$$
\begin{aligned}
& \mathrm{s}(\theta)=\operatorname{Sin}(\theta), \mathrm{S} \phi=\operatorname{Sin}(\phi), \mathrm{S}(\psi)=\operatorname{Sin}(\psi) \\
& \mathrm{c}(\theta)=\operatorname{Cos}(\theta), C \phi=\operatorname{Cos}(\phi), C(\psi)=\operatorname{Cos}(\psi)
\end{aligned}
$$

Table 2: Design Parameters And Its Specification

\begin{tabular}{|l|l|l|}
\hline S. No & Parameters & Specification \\
\hline 1. & Supply voltage & $3.2-5 \mathrm{~V}$ \\
\hline 2. & TX/RX Frequency & $2.4 \mathrm{GHz} / 5.8 \mathrm{GHz}$ \\
\hline 3. & Satellite Positioning & $\mathrm{GPS}$ \\
\hline 4. & Cost & low \\
\hline 5. & Flight Time & High \\
\hline 6. & Weight & $350 \mathrm{~g}$ \\
\hline 7. & Sensor & Ultrasonic \\
\hline 8. & Video Modes & Available \\
\hline 9. & Coverage & Large \\
\hline 10. & take off/ landing Area & Very Small \\
\hline 11. & Battery & Lipo Battery \\
\hline 12. & Maximum flight speed & $16 \mathrm{~m} / \mathrm{s}$ \\
\hline 13. & Android App & Available \\
\hline
\end{tabular}

\section{Conclusion and Future Scope}

The major aim of our project is to study the complete design and flight dynamics of quad copter from the engineering perspective and to create an enhanced working model of UAV with significant improvement in obstacle detection and control with the help of ultrasonic eco sensors which makes the design very unique. Our main objective was to construct a UAV which can be used for multipurpose applications in military, spyware, industrial and commercial applications like monitoring and management of traffic, rescue and searching operations, altitude estimation, capturing of remote images in various terrains, collecting data from nuclear radioactive hazard prone areas, Locating frost conditions, fire detection in farmlands and identification of objects. Our quad copter has been successfully designed, implemented and tested for all conditions. It can work with high accuracy, precision and control providing stability to the hovering action of UAV. On the basis of test results GUI controller from remote controlling device, we can send the control signal to the flight connector board for providing good balance and stability providing optimal performance creating a benchmark in quad copter design and control technology. With the help of our innovative novel technical design, we can implement lot of data capturing, processing and transmission . We chose the java based graphical and voice processing module to enable easy control of the UAV and simplicity of design, good flexibility, portability with vast opportunity and significant potential of future research in the field of aerodynamics engineering. This project can be enhanced further in various research works to amalgamate various technologies with UAVs to get various useful outputs augmenting the efficiency and performance of the quad copter.

\section{References}

[1] Mohd Khan, Quad copter Flight Dynamics, International journal of scientific \& technology research vol.3 issue 8 august 2014.

[2] Ankyda Ji, Kamran Turkoglu, , "Development of a Low-Cost Experimental Quadcopter Testbed Using an Arduino Controller and Software,"

[3] Mori, T., Nonaka, T., \& Hase, T. (2010, October). Design method of GUI using genetic algorithm. In Systems Man and Cybernetics (SMC), 2010 IEEE International Conference on (pp. 3200-3204). IEEE.

[4] Rauf, Abdul, Sajid Anwar, and M. Arfan Jaffer. "Automated GUI test coverage analysis using GA." Information Technology: New Generations (ITNG), 2010 Seventh International Conference on. IEEE, 2010. 
[5] L. Sun, H. Xie and K. Chen, "Design and Realization of GUI-Control for Leak Detection Equipment," Proceeding of the International Conference on Electronic, Communi- cation and Control (ICECC), 9-11 September 2011, pp. 3638-3641

[6] D. Park, M.-S. Park and S.-K. Hong, "A Study on the 3-DOF Attitude Control of Free-Flying Vehicle," Pro-ceeding of the IEEE International Symposium on Indus-trial Electronics (ISIE), Pusan, 12-16 June 2001, Vol. 2, pp. 1260-1265.

[7] A. A. Mian and W. Daobo, "Nonlinear Flight Control Strategy for an Underactuated quadrotor aerial robot." Proceeding of the IEEE International Conference on Networking, Sensing and Control (ICNSC), Sanya, 6-8 April 2008, pp. 938-942.

[8] M. Achtelik, T. Zhang, K.Kuhnlenz and M. Buss, "Vis-ual Tracking and Control of a Quadcopter Using a Stereo Camera System and Inertial Sensors," Proceeding of the International Conference on Mechatronics and Automa-tion (ICMA), 9-12 August 2009, pp. 2863-2869.

[9] M. Santos, V. López and F. Morata, "Intelligent Fuzzy Controller of a Quadrotor," Proceeding of the IEEE In-ternational Conference on Intelligent Systems and Know- ledge Engineering (ISKE), Hangzhou, 15-16 November 2010, pp. 141-146.

[10] I. Morar and I. Nascu, "Model Simplification of an Un-manned Aerial Vehicle," Proceeding of the IEEE Interna-tional Conference on Automation Quality and Testing Robotics (AQTR), Cluj Napoca 24-27 May 2012 pp. 591-596.

[11] J. Li and Y. T. Li, "Dynamic Analysis and PID Control for a Quadrotor," Proceeding of the International Conference on Mechatronics and Automation (ICMA), Bei-jing, 7-10 August 2011, pp.573-678.

[12] Duckgee Park; Moon-Soo Park; Suk-Kyo Hong, "A study on the 3-DOF attitude control of free-flying vehicle," in Industrial Electronics, 2001. Proceedings. ISIE 2001. IEEE International Symposium on , vol.2, no., pp.1260-1265 vol.2, 2001

[13] Morar, I.; Nascu, I., "Model simplification of an unmanned aerial vehicle," in Automation Quality and Testing Robotics (AQTR), 2012 IEEE International Conference on , vol., no., pp.591-596, 24-27 May 2012

[14] Achtelik, M.; Tianguang Zhang; Kuhnlenz, K.; Buss, M., "Visual tracking and control of a quadcopter using a stereo camera system and inertial sensors," in Mechatronics and Automation, 2009. ICMA 2009. International Conference on , vol., no., pp.2863-2869, 912 Aug. 2009

[15] Miguel A. Olivares-Mendez, Luis Mejias, Pascual Campoy, Ignacio Mellado-Bataller "Cross-Entropy Optimization for Scaling Factors of a Fuzzy Controller: A See-and-Avoid Approach for Unmanned Aerial Systems Journal of Intelligent \& Robotic Systems January 2013, Volume 69, Issue 1, pp 189-205 